\title{
Factors Influencing Health Workers' Compliance with the WHO Intermittent Preventive Treatment for Malaria in Pregnancy Recommendations in the Northern Region, Ghana
}

Abdul Gafaru Mohammed

University of Ghana School of Public Health

Dwomoh Duah

University of Ghana School of Public Health

Ernest Kenu

University of Ghana School of Public Health

Justice Nonvignon

University of Ghana School of Public Health

Alex Manu

University of Ghana School of Public Health

Harriet Affran Bonful ( $\square$ habonful@ug.edu.gh )

University of Ghana School of Public Health https://orcid.org/0000-0003-0069-3241

\section{Research Article}

Keywords: Compliance, Health workers, IPTp-SP, Ghana, Northern Region, Malaria, Adherence

Posted Date: December 13th, 2021

DOI: https://doi.org/10.21203/rs.3.rs-1129464/v1

License: (c) (1) This work is licensed under a Creative Commons Attribution 4.0 International License. Read Full License 


\section{Abstract \\ Background}

Although IPTp-SP is a lifesaving WHO recommended preventive intervention for pregnant women in malariaendemic regions, IPTp-SP uptake in the Northern region of Ghana is much lower than the sub-optimal national coverage level. Assessing the extent of health workers' compliance and its associated factors will generate valuable pointers to be targeted at the program level. The study examined the factors influencing health workers' compliance with the WHO recommended guidelines for IPTp-SP in the Northern Region.

\section{Methods}

A cross-sectional study among 315 health workers in the Northern region was conducted. Semi-structured questionnaires were used to collect data on health workers' sociodemographic characteristics, facility-based factors and knowledge level. Data were collected on health workers' compliance with the recommended practices through covert observations using a checklist. Facility observations were carried out using a checklist. Crude and adjusted logistic regression were used to determine predictors of health workers' compliance, at $5 \%$ significance level adjusting for clustering.

\section{Results}

Of the 315 health workers studied, the median age was 29 years ( $26-34$ years). Females constituted 252 (80.5\%) of the 313 workers. The majority $148(47.44 \%)$ of the 312 health workers were midwives. Overall, $56.2 \%$ (CI $51.0-$ 62.0) were adequately complying with the recommended guidelines. Lower levels of compliance were recorded in health centers $15.6 \%$ (5.0 - 33.0) and CHPS compounds $21.2 \%(11.0-35.0)$. The factors associated with compliance included health workers' knowledge (aOR $=7.64,95 \% \mathrm{Cl} 4.21-13.87, \mathrm{p}<0.001)$, job satisfaction $(\mathrm{aOR} 10.87,95 \% \mathrm{Cl}$ 7.04 - 16.79, $p<0.001$ ), in-service training (aOR $10.11,95 \% \mathrm{Cl} 4.53-22.56, \mathrm{p}<0.001)$, supervision (aOR $4.01,95 \% \mathrm{Cl}$ $2.09-7.68, p<0.001$ ), availability of job aids (aOR $3.61,95 \% \mathrm{Cl} 2.44-5.35, p<0.001)$, health workers experience (aOR $=10.64,95 \% \mathrm{Cl} 5.99-18.91, \mathrm{p}<0.001)$ and facility type (aOR 0.03, 95\% Cl $0.01-0.07, p<0.001)$.

\section{Conclusion}

Compliance with the recommended IPTp-SP guidelines is suboptimal in the region, with lower-level health facilities recording the least compliance levels. Health centers and CHPS facilities should be prioritized in distributing limited resources to improve health worker quality of care for antenatal care clients.

\section{Background}

Malaria in pregnancy is a significant public health problem. In 2018, an estimated 11 million pregnant women living in 38 countries with moderate-to-high malaria transmission in Sub-Saharan Africa were diagnosed with malaria ${ }^{1}$. Malaria in pregnancy accounts for $16.8 \%$ of all hospital admissions and $3.4 \%$ of deaths, making it the singular highest contributor to all OPD admissions among pregnant women in Ghana ${ }^{2,3}$. 
To reduce the morbidity and mortality associated with malaria, the World Health Organization (WHO) recommended the use of the Intermittent Preventive Treatment for malaria in pregnancy using sulfadoxine-pyrimethamine (IPTpSP) in 2000 alongside other preventive and curative interventions. The IPTp-SP protects between 65 and $85 \%$ of pregnant women from placental malaria infection ${ }^{4}$. The WHO updated the recommendations for IPTp-SP in 2012. The updated three or more doses of IPTp-SP has been demonstrated to be associated with an enhanced reduction in maternal parasitemia, malaria-related maternal anaemia and poor birth outcomes ${ }^{5-10}$. Per the updated guideline, IPTp-SP should be commenced early in the second trimester, with doses given at least one month apart under direct observation until delivery ${ }^{6}$. Subsequently, the Ghana National Malaria Control Programme revised the national IPTpSP policy from up to three doses of IPTp-SP to three or more doses until delivery in $2014^{11}$. The three or more IPTpSP doses are linked with a greater mean birth weight and fewer low birth weight (LBW) newborns than the two doses 12 .

However, two-thirds of pregnant women do not receive the recommended IPTp-SP doses during their visits to ANC units in Africa ${ }^{13}$. Data from cross-sectional surveys demonstrate that despite the availability of SP, eligible women accessing ANC services in the Volta and Northern regions of Ghana were not offered SP dose ${ }^{14,15}$. Additionally, although the national target for IPTp-SP3 uptake is $80.0 \%$, data from the District Health Information Management System II (DHIMS II) at the national level indicates that IPTp-SP3 uptake has been below 49\% since 2017 16,17. In fact, the Northern region of Ghana recorded a more significant decline in uptake from $36.4 \%$ in 2019 to $27.2 \%$ in $2020^{16}$.

Health workers' inappropriate delivery of IPTp-SP services is a threat to reducing maternal malaria, maternal deaths, and poor birth outcomes. According to the WHO, an essential factor for the low uptake of IPTp-SP3 among pregnant women is confusion among health workers about the IPTp-SP recommendations ${ }^{6}$. In a national survey conducted in 2019 , the commonly cited reasons for which $39.0 \%$ of pregnant did not receive the required dose during their most recent pregnancy were that they were not aware they had to take more (42\%), and health workers did not give it to them $(35 \%)^{18}$.

A combination of interventions is required to improve health workers' compliance with the WHO IPTp-SP recommendation. These include; regular in-service training, monitoring, supportive supervision, career development programs, providing logistics (portable water, cups for DOT practice, SP drug, and IPT manuals o leaflets). Reducing staff workload and motivating staff are equally important conditions worth addressing 15,19,20.

Measuring health workers' compliance with the revised IPTp-SP guidelines and the associated factors will enhance the early identification of areas that need the attention of policymakers and implementors. Researchers can create a composite compliance indicator using 7 binary questions-items ${ }^{19}$. In an attempt to examine the problem of poor compliance to the recommended IPTp-SP guidelines in Ghana, most of the studies considered less than the ideal number of question-items of the IPTp-SP guideline in assessing health workers compliance, potentially leading to an underestimation of compliance level and thus masking the actual burden ${ }^{15,20-23}$. Therefore, this study sought to generate data on health workers compliance considering all components of the IPTp-SP guideline and the factors associated in the Northern region.

\section{Methods}

\section{Study design and Setting}


We conducted a cross-sectional study among antenatal health workers in the Northern Region of Ghana from April to July 2021. The Northern Region is one of the sixteen regions of Ghana. The annual temperature range is between 25 and $30^{\circ} \mathrm{C}$, which is favourable for Anopheles larval development. The region has 308 registered health facilities offering antenatal care services. The region has an estimated 3346 registered community health nurses, enrolled nurses, professional nurses and midwives. Each month, hospitals and polyclinics obtain their supplies of sulphadoxine-pyrimethamine for IPTp administration from the Northern Regional medical stores at no cost. Health centers and CHPs at the district level depend on the district medical stores for SP supplies. According to the 2019 Malaria Indicator Survey, $64.5 \%$ of pregnant women in the region received three or more doses of IPTp-SP between 2017 - 2019, although $91.8 \%$ ANC attendance was recorded ${ }^{18}$.

\section{Study Eligibility and Sampling Process}

ANC staff who had worked for at least one year at the selected health facilities and were present at the study time were eligible. Those who had worked for at least one year but were not directly involved in IPTp-SP administration were excluded.

We employed a multistage sampling approach in the sampling of 286 health workers. The first stage involved a simple random sampling of 8 districts from the 16 districts in the region. The next stage involved using a restricted stratified sampling approach to select 16 health facilities from the sampled eight districts. In each district, we divided the health facilities into stratum A (district hospitals) and stratum B (Community-based Health Planning and Services (CHPS) compounds, health centres, polyclinics). One facility was selected from each stratum, making up the two health facilities in the district. The last stage involved sampling of 286 health workers from the 16 health facilities sampled. We used a proportionate to size sampling approach to determine the number of health workers to sample from each health facility. Simple random sampling was then used to sample health workers at the various facilities on survey days.

\section{Data Collection and Study Variables}

Data was consecutively collected in two phases. During the first phase, semi-structured questionnaires were administered to the sampled health workers. The questionnaire collected information on health workers' sociodemographic and individual-based factors. Knowledge was measured using an 11 question item tool for assessing health workers knowledge by Oluwasomidoyin, Bello, \& Oni, (2020) (Supplement 1). The second phase involved the collection of data on the primary outcome; health workers compliance with the revised IPTp-SP guidelines. This data was collected by covertly observing how health workers performed 7 activities prescribed in the revised guidelines (Supplement 2). Each research assistant was responsible for observing the IPTp-SP practices of their respective respondents. The research assistants observed each of the respondents as they administered the services to their clients. Finally, using a checklist, each health facility was assessed for the availability of SP, portable water and IPTp-SP posters (Supplement 3).

\section{Data Management and Statistical Analysis}

The returned questionnaires were cleaned and edited to ensure accuracy and completeness before coding in Microsoft Excel 2017 and analyzing using STATA version 15.0 (StataCorp LLC). We excluded all forms with missing data on the primary outcome from the analysis $(n=15)$. Regarding the dependent variable, a correct practice was scored 1 , and a wrong practice scored 0 . We categorized the total scores obtained by each health worker into inadequate compliance (0-3) and adequate compliance (4-7) ${ }^{25}$. Each correct response to the knowledge question 
items was scored 1, and an incorrect response scored 0 . We categorized the scores into low knowledge (0-4), moderate knowledge (5-8) and high knowledge (9-11) ${ }^{24}$. All categorical variables comprising age, sex, cadre, level of education, length of practice, awareness and level of knowledge, type of facility, monitoring and supervision, staff workload, routine training, IPT job aids and manuals, and staff motivation were analyzed into frequencies and proportions at $95 \% \mathrm{Cl}$. Skewed continuous variable, for example, age was presented using a median and interquartile range.

We performed a crude logistic regression analysis to test the association between the independent variables and compliance.

We further selected variables with statistically significant association with the health workers' compliance at a significance level of 0.1 for an adjusted logistic regression. Robust standard errors were used to adjust for clustering in both the crude and adjusted analyses, with the type of facility as the main clustering variable. A significance level of $5 \%$ was set for the adjusted logistic analysis. Findings from the health facility assessments were analyzed into frequencies and proportions.

\section{Results}

\section{Health Workers Background Characteristics}

Of the 315 health care workers studied, more than two thirds $252(80.5 \%)$ were females. The median age of the health workers was 29 years (26 - 34 years). On education, $149(48.7 \%)$ of the 306 health workers studied were diploma holders, whiles 53 (17.3\%) were degree holders. The majority 177 (59.2\%) of the 299 health workers studied had been providing obstetric care for less than five years (Table 1)

Table 1: Background Characteristics of Health Workers studied, Northern Region, 2021 


\begin{tabular}{|c|c|c|}
\hline Characteristics & Frequency $(\mathrm{N}=315)$ & Percentage (\%) \\
\hline \multicolumn{3}{|l|}{ Sex } \\
\hline Male & 61 & 19.5 \\
\hline Female & 252 & 80.5 \\
\hline Subtotal & 313 & 100.0 \\
\hline \multicolumn{3}{|l|}{ Experience } \\
\hline$>5$ & 177 & 59.2 \\
\hline $5-10$ & 72 & 24.1 \\
\hline $10+$ & 50 & 16.7 \\
\hline Subtotal & 299 & 100.0 \\
\hline \multicolumn{3}{|l|}{ Cadre } \\
\hline General nurse & 124 & 39.7 \\
\hline Midwife & 148 & 47.5 \\
\hline Others & 40 & 12.8 \\
\hline Subtotal & 312 & 100.0 \\
\hline \multicolumn{3}{|l|}{ Education } \\
\hline Certificate & 104 & 34.0 \\
\hline Diploma & 149 & 48.7 \\
\hline Degree & 53 & 17.3 \\
\hline \multirow[t]{2}{*}{ Subtotal } & 306 & 100.0 \\
\hline & Median (years) & IQR (years) \\
\hline Age of HWs & 29.0 & $26.0-34.0$ \\
\hline
\end{tabular}

\section{Health Workers' Compliance with the IPTp-SP Recommended Treatment Guidelines}

Majority 231 (73.3\%) of the 315 health workers properly documented the IPTp-SP services rendered in patients' ANC cards. Also, $228(72.4 \%)$ of the 315 health workers studied did not ask if pregnant women were on other medications for example co-trimoxazole. On informing pregnant women on the next dose's date, 179 (56.8\%) of the 315 health workers failed to inform the pregnant women they attended (Table 2).

Table 2: Observation of Antenatal Health Workers IPTp-SP practices, Northern Region, 2021 (N=315) 


\begin{tabular}{|c|c|c|}
\hline Compliance to IPTp treatment guidelines & Frequency $(\mathrm{N})$ & Percentage (\%) \\
\hline \multicolumn{3}{|l|}{ Confirmed gestational period } \\
\hline Yes & 210 & 66.7 \\
\hline No & 105 & 33.3 \\
\hline \multicolumn{3}{|l|}{ Report adverse reactions } \\
\hline Yes & 110 & 34.9 \\
\hline No & 205 & 65.1 \\
\hline \multicolumn{3}{|l|}{ Proper documentation } \\
\hline Yes & 231 & 73.3 \\
\hline No & 84 & 26.7 \\
\hline \multicolumn{3}{|l|}{ DOT practice } \\
\hline Yes & 277 & 87.9 \\
\hline No & 38 & 12.1 \\
\hline \multicolumn{3}{|l|}{ Other medications (co-trimoxazole) } \\
\hline Yes & 87 & 27.6 \\
\hline No & 228 & 72.4 \\
\hline \multicolumn{3}{|l|}{ Required dosage } \\
\hline Yes & 306 & 97.1 \\
\hline No & 9 & 2.9 \\
\hline \multicolumn{3}{|l|}{ Next dose } \\
\hline Yes & 136 & 43.2 \\
\hline No & 179 & 56.8 \\
\hline
\end{tabular}

\section{Stratification of Health Workers' Compliance level by Health Facility Type}

Overall, the majority $56.2 \%$ (CI 51.0 - 62.0) of the health workers complied with the recommended IPTp-SP practices adequately. However, lower levels of compliance were recorded in the health centers and CHPS compounds (Table 3).

Table 3: Stratification of Health Workers' Compliance level by Health Facility type, Northern Region, 2021 


\begin{tabular}{|lll|}
\hline Facility type & Compliance level (\%) & $95 \% \mathrm{Cl}$ \\
\hline CHPS & 21.20 & $11.0-35.0$ \\
\hline Health Centre & 15.60 & $5.0-33.0$ \\
\hline Hospital & 69.70 & $63.0-76.0$ \\
\hline
\end{tabular}

\section{Association between Health worker-related factors and their Compliance with IPTp-SP guidelines}

From the multivariable regression analysis, health workers' experience, knowledge and use of manuals from personal sources were significantly associated with their compliance with the WHO IPTp-SP guidelines. Health workers who personally sourced and read IPTp-SP manuals had 1.9 times odds of compliance with the recommended guidelines compared to their counterparts ( $\mathrm{aOR}=1.89,95 \% \mathrm{Cl} 1.04-3.43, \mathrm{p}<0.036)$. Similarly, the odds of complying with the recommended guidelines was 2.8 times among health workers with $5-10$ years working experience $(\mathrm{aOR}=2.78,95 \% \mathrm{Cl} 1.06-7.24, \mathrm{p}<0.037)$ and 10.6 times among those with more than ten years of working experience $(\mathrm{aOR}=10.64,95 \% \mathrm{Cl} 5.99-18.91, \mathrm{p}<0.001)$ compared with health workers with less than five years working experience.

Regarding the knowledge level of the health workers, the odds of complying with the recommended guidelines was 3.2 times among health workers with moderate knowledge compared to those with low knowledge $(\mathrm{aOR}=3.20,95 \%$ $\mathrm{Cl} 2.28$ - 4.49, $\mathrm{p}<0.001)$. Also, the odds of complying with the recommended guidelines was 7.6 times among health workers with high knowledge compared to those with low knowledge of the guidelines ( $\mathrm{aOR}=7.64,95 \% \mathrm{Cl} 4.21$ 13.87, p<0.001) (Table 4)

Table 4: Association between Health worker-related factors and their Compliance with IPTp-SP guidelines in the Northern Region 


\begin{tabular}{|c|c|c|c|c|c|c|}
\hline \multirow{2}{*}{$\begin{array}{l}\text { Variable } \\
\text { Age of HWs }\end{array}$} & \multicolumn{2}{|c|}{ cOR (95 \% C.I) } & \multirow{2}{*}{$\begin{array}{l}\text { P-value } \\
0.001\end{array}$} & \multicolumn{2}{|c|}{ aOR (95 \% C.I) } & \multirow{2}{*}{$\begin{array}{l}\text { P-value } \\
0.325\end{array}$} \\
\hline & $1.11(1.1$ & 1.16) & & 0.99 (0.96 & 1.01) & \\
\hline \multicolumn{7}{|l|}{ Sex } \\
\hline Male & 1.00 & & & & & \\
\hline Female & $0.85(0.48$ & 1.51) & 0.586 & $0.52(0.15$ & 1.79) & 0.302 \\
\hline \multicolumn{7}{|l|}{ Experience } \\
\hline$<5$ & 1.00 & & & & & \\
\hline $5-10$ & $3.23(1.78$ & $5.85)$ & 0.001 & $2.78(1.06$ & 7.24) & $0.037^{++}$ \\
\hline$>10$ & $5.65(2.59$ & 12.33) & 0.001 & $10.64(5.99$ & 18.91) & $0.001^{++}$ \\
\hline \multicolumn{7}{|l|}{ Cadre } \\
\hline General Nurse & 1.00 & & & & & \\
\hline Midwife & $1.66(1.02$ & 2.68) & 0.041 & $0.15(0.06$ & $0.37)$ & 0.001 \\
\hline Others & $1.98(0.95$ & $4.15)$ & 0.070 & $1.34(0.65$ & $2.78)$ & 0.429 \\
\hline \multicolumn{7}{|l|}{ Education } \\
\hline Certificate & 1.00 & & & & & \\
\hline Diploma & $1.36(0.82$ & $2.24)$ & 0.232 & $2.37(0.99$ & $5.68)$ & 0.052 \\
\hline Degree & $3.69(1.74$ & 7.81) & 0.001 & $2.27(0.71$ & 7.26) & 0.165 \\
\hline \multicolumn{7}{|l|}{ Awareness } \\
\hline Not Aware & 1.00 & & & & & \\
\hline Aware & $1.51(0.91$ & 2.48) & 0.108 & $2.56(0.86$ & 7.78) & 0.092 \\
\hline \multicolumn{7}{|l|}{ Knowledge level } \\
\hline Low & 1.00 & & & & & \\
\hline Moderate & $2.90(1.17$ & 7.17) & 0.021 & $3.20(2.28$ & 4.49) & $0.001^{++}$ \\
\hline High & $9.21(3.54$ & 23.96) & 0.001 & $7.64(4.21$ & 13.87) & $0.001^{++}$ \\
\hline \multicolumn{7}{|c|}{ Access to IPTp materials } \\
\hline Not Accessed & 1.00 & & & & & \\
\hline Accessed & $2.21(1.39$ & $3.51)$ & 0.001 & $1.89(1.04$ & $3.43)$ & $0.036^{++}$ \\
\hline
\end{tabular}

++statistically significant

\section{Association between Facility-based factors and Health Workers' Compliance Level}


Facility type, job training, staff motivation, supervision and availability of IPTp-SP job aids and manuals were significantly associated with health workers compliance. Health workers who received in-service training on the revised IPTp-SP guidelines had 10.0 times odds of complying with the guidelines compared with those who had not received any training on IPTp-SP administration (aOR 10.11, 95\% Cl $4.53-22.56, p<0.001)$.

The odds of compliance among health workers satisfied with the ANC unit's conditions were 10.9 times compared with those not satisfied with the conditions of their working environment (aOR 10.87, 95\% Cl 7.04 - 16.79 p<0.001).

Similarly, health workers who had been supervised had 4.0 times odds of compliance with the recommended practices than those who had not received any supervisory visits (aOR 4.01, 95\% Cl 2.09 - 7.68, p<0.001). Health workers who received IPTp-SP training manuals from their facilities had 3.6 times odds of compliance compared with their counterparts who did not receive these materials from their facilities (aOR 3.61, 95\% $\mathrm{Cl} 2.44-5.35$, $\mathrm{p}<0.001$ ) (Table 5).

Table 5: Association between facility-based factors and health workers' Compliance with IPTp-SP guidelines, Northern Region 


\begin{tabular}{|c|c|c|c|c|c|c|}
\hline Variable & \multicolumn{2}{|c|}{$\operatorname{cOR}(95 \% \mathrm{Cl})$} & P-value & \multicolumn{2}{|c|}{ aOR $(95 \% \mathrm{Cl})$} & P-value \\
\hline \multicolumn{7}{|l|}{ Facility type } \\
\hline Hospital & 1.00 & & & & & \\
\hline Health Centre & $0.08(0.03$ & $0.22)$ & 0.001 & $0.03(0.01$ & $0.07)$ & $0.001^{++}$ \\
\hline CHPS compound & $0.11(0.06$ & $0.24)$ & 0.001 & $0.03(0.02$ & $0.05)$ & $0.001^{++}$ \\
\hline \multicolumn{7}{|l|}{ Staff workload } \\
\hline $0-30$ & 1.00 & & & & & \\
\hline$>30$ & $0.95(0.60$ & 1.52) & 0.829 & - & & - \\
\hline \multicolumn{7}{|l|}{ Training } \\
\hline No & 1.00 & & & & & \\
\hline Yes & $13.11(7.60$ & 22.62) & 0.001 & $10.11(4.53$ & $22.56)$ & $0.001^{++}$ \\
\hline \multicolumn{7}{|l|}{ Monitoring } \\
\hline No & 1.00 & & & & & \\
\hline Yes & $16.63(9.21$ & $30.02)$ & 0.001 & $4.01(2.09$ & 7.68) & $0.001^{++}$ \\
\hline \multicolumn{7}{|l|}{ Job satisfaction } \\
\hline Not satisfied & 1.00 & & & & & \\
\hline Satisfied & $12.24(7.04$ & $21.26)$ & 0.001 & $10.87(7.04$ & 16.79) & $0.001^{++}$ \\
\hline \multicolumn{7}{|l|}{ IPT training manual } \\
\hline Not provided & 1.00 & & & & & \\
\hline Provided & $4.50(2.55$ & 7.95) & 0.001 & $3.61(2.44$ & $5.35)$ & $0.001^{++}$ \\
\hline \multicolumn{7}{|l|}{ Shortage of SP } \\
\hline No & 1.00 & & & & & \\
\hline Yes & $0.42(0.25$ & $0.71)$ & 0.001 & $1.08(0.71$ & 1.64) & 0.730 \\
\hline
\end{tabular}

++statistically significant

\section{Health facility IPTp-SP implementation assessment}

Health education programs on malaria in pregnancy were available in $75.0 \%(12 / 16)$ of the facilities visited. The majority $62.5 \%(10 / 16)$, of health facilities visited had their quarterly education programs, including IPTp-SP. Health talks were delivered in 8 of the 16 health facilities visited. Of these eight facilities, five included malaria in pregnancy in their presentation, whereas only three mentioned IPTp-SP in their presentation. SP was available in all the facilities visited, with eight facilities having posters of IPTp-SP pasted on the walls of the ANC unit. DOT was practiced in all the facilities visited; however, only two of these health facilities had potable water available for pregnant women. 
Pregnant women bought water from a seller in the ANC unit or a nearby provision store in the remaining 14 health facilities.

\section{Discussion}

The study indicates that health workers' experience, knowledge level, training on IPTp-SP, job satisfaction, monitoring, type of facility they work in, and availability of IPTp-SP Job aids were significantly associated with health workers' appropriate delivery of IPTp-SP. Our study used a non-participant observation design, which allowed the identification of health workers compliance with the IPTp-SP recommended guidelines and eliminated the possibility of Hawthorne's effect.

Overall, low compliance by health workers recorded at all health system levels was more pronounced in the health centers and CHPS compounds. These CHPS compounds and health centers serve as the first point of health care for most pregnant women in the villages. If not addressed, the high inappropriate delivery of IPTp-SP services will curtail the impact of all demand-side related measures taken to address the low uptake of IPTp-SP in the region. More costeffective measures adopted to address low compliance in the region should be targeted at the health centres and CHPS compounds. The observed high inadequate compliance among health workers from the CHPS compounds and health centers corroborates the results of a study in the Volta region of Ghana, where inappropriate delivery of IPTp-SP and ITN services were recorded in all facilities studied and more pronounced in the lower level of health care 14. The consistency in findings could be attributed to the use of observations to assess IPTp-SP delivery in both studies though, De-gaulle \& Kamgno used a three-indicator algorithm to assess health workers' compliance compared to the seven-indicator algorithm employed in the present study.

In-service training of Antenatal health care workers keeps them informed and technically equipped to deliver IPTp-SP services to pregnant women. Despite its relevance, training is sometimes overlooked, affecting health workers' perception of IPTp-SP and its administration. The increased odds of compliance among trained health workers in this study further substantiate the relevance of training to frontline health workers. The significance of training on health workers' compliance with appropriate practices has also been revealed in other studies among African countries ${ }^{26,27}$. To ensure adequate training of health workers, the NMCP needs to move from the cascade form of training and adopt a more peripheral system of training where health workers at the health centers and CHPS compounds benefit from the training.

Supervision and monitoring are necessary to keep frontline health workers on their toes and to make them comply with the recommended guidelines. Health workers who had been supervised had an increased odds of compliance with the recommended guidelines. The finding is consistent with a study conducted in Uganda where health workers' uncertainty of SP and the new IPTp-SP guidelines was associated with a lack of supervision ${ }^{28}$. This implies that an increase in supervision might increase the appropriate delivery of IPTp-SP services in the region. The Onsite Training and Supportive Supervision (OTSS) adopted by the National Malaria Control Program and IMPACT MALARIA for other malaria control areas such as case management and laboratory diagnosis could be extended to the delivery of IPTp-SP services.

Nevertheless, in a study conducted by Maheu-Giroux \& Castro (2014), health care staff supervised during the six months prior to the study were less likely to deliver IPTp during a consultation than those not supervised. The study, unlike this one adopted a secondary data analysis design which could have accounted for the inconsistency in findings.

Page $12 / 16$ 
Health workers access to IPTp training manuals and job aids keep them informed on relevant changes made to existing guidelines. Heath workers with access to job aids and training materials had increased odds of complying with the recommended guidelines. This is similar to the findings of a study conducted among five African countries where IPTp guidelines at health facilities was a significant determinant of IPTp-SP appropriate delivery ${ }^{29}$.

Health workers are supposed to be motivated to perform IPTp-SP services to the best of their abilities. However, motivating health workers has not been given the needed attention it deserves as a factor that can influence all forms of health service deliveries. In a study conducted in Tanzania, about $80.0 \%$ of health workers expressed dissatisfaction as a constrain to their performance ${ }^{30}$. Health workers who were satisfied with the ANC unit's conditions had increased odds of compliance. Incentivizing best performing health workers at the ANC units of health facilities will motivate health workers to deliver IPTp-SP services.

A few limitations were, however, identified in the study design used. The study used covert observation to determine whether health workers were complying with all indicators of the recommended guidelines. One indicator, the proper documentation of services rendered could not be reported based on the observation in a few cases. Research assistants engaged ANC clients to confirm proper IPTp-SP services in their ANC booklets to curtail this limitation. Before the actual data collection, the research assistants received thorough training on data collection tools and extensive pre-test activities to ensure that they were able to gather the data accurately by observing and listening intently to the delivery of care by ANC health workers.

\section{Conclusion}

Compliance with the recommended IPTp-SP guidelines is suboptimal in the region, with lower health facilities recording the least compliance levels. Health centers and CHPS facilities should be prioritized in the distribution of limited resources to improve health worker quality of care for antenatal care clients.

\section{List Of Abbreviations}
ANC
Antenatal Care
CHPS
Community based Health Planning and Services
DHIMS
District Health Information Management System
DOT
Direct Observation Therapy
GFELTP
Ghana Field Epidemiology and Laboratory Training Programme
IPTp-SP
Intermittent Preventive Treatment for Malaria in Pregnancy using
Sulfadoxine Pyrimethamine
NMCP National Malaria Control Programme
OPD Outpatient Department
OTSS Onsite Training and Supportive Supervision
WHO World Health Organization

Page 13/16 


\section{Declarations}

\section{Ethical declarations}

The Ethical Review Committee of the Ghana Health Service approved the protocol for the study with ethics approval number GHS-ERC 032/05/21. The Northern Regional Health Directorate and heads of the various health facilities provided administrative authorization. We obtained written informed consent from all the health workers.

\section{Consent for publication}

Not applicable

\section{Availability of dataset or materials}

The datasets used and/or analyzed during the current study are available from the corresponding author on reasonable request.

\section{Competing interest}

The authors declare that they have no competing interest

\section{Funding}

Not applicable

\section{Contributions}

AGM conceptualized the study, collected data and drafted the initial manuscript. HAB and AM supervised the entire research project. DD, JN, EK and AGM, and HAB, performed the analysis and interpreted the results. All authors reviewed, edited and approved the final manuscript for submission.

\section{Acknowledgement}

We are grateful to the National Malaria Control Programme (NMCP), the Ghana Field Epidemiology and Laboratory Training Programme (GFELTP) and the Northern Regional Health Directorate for their support. We acknowledge all the health workers who participated in the study and the field team.

\section{References}

1. WHO. The "World malaria report 2019" at a glance [Internet]. 2019 [cited 2020 Oct 17]. Available from: https://www.who.int/news-room/feature-stories/detail/world-malaria-report-2019

2. NMCP. MEDBOX | 2014 Annual Report: National Malaria Control Programme [Internet]. 2014 [cited 2020 Oct 13]. Available from: https://www.medbox.org/document/2014-annual-report-national-malaria-controlprogramme\#GO

3. Owusu-Boateng I, Anto F. Intermittent preventive treatment of malaria in pregnancy: a cross-sectional survey to assess uptake of the new sulfadoxine-pyrimethamine five dose policy in Ghana. Malar J [Internet]. 2017 Aug 10 [cited 2020 Oct 13];16(1):323. Available from:

http://malariajournal.biomedcentral.com/articles/10.1186/s12936-017-1969-7 
4. Garner P, Am G. Drugs for preventing malaria in pregnant women (Review). 2009 [cited 2021 Oct 29]; Available from: http://www.thecochranelibrary.com

5. Amoakoh-Coleman M, Arhinful DK, Klipstein-Grobusch K, Ansah EK, Koram KA. Coverage of intermittent preventive treatment of malaria in pregnancy (IPTp) influences delivery outcomes among women with obstetric referrals at the district level in Ghana. Malar J 2020191 [Internet]. 2020 Jun 24 [cited 2021 Nov 1];19(1):1-13. Available from: https://malariajournal.biomedcentral.com/articles/10.1186/s12936-020-03288-4

6. WHO Global Malaria Programme. New WHO recommendations for IPTp-SP [Internet]. 2013 [cited 2020 Oct 17]. Available from: http://whqlibdoc.who.int/publications/2010/9789241599412_eng.pdf

7. Orobaton N, Austin AM, Abegunde D, Ibrahim M, Mohammed Z, Abdul-Azeez J, et al. Scaling-up the use of sulfadoxine-pyrimethamine for the preventive treatment of malaria in pregnancy: results and lessons on scalability, costs and programme impact from three local government areas in Sokoto State, Nigeria. Malar J [Internet]. 2016 Nov 4 [cited 2021 Nov 1];15(1):1-24. Available from:

https://pubmed.ncbi.nlm.nih.gov/27814763/

8. Dapaa S. Uptake of Intermittent Preventive Treatment for Malaria and Birth Outcomes in Selected Health Facilities in the Brong Ahafo Region of Ghana. 2017;(10551624):1-77.

9. Gutman J, Mwandama D, Wiegand RE, Ali D, Mathanga DP, Skarbinski J. Effectiveness of intermittent preventive treatment with sulfadoxine-pyrimethamine during pregnancy on maternal and birth outcomes in Machinga District, Malawi. In: Journal of Infectious Diseases [Internet]. J Infect Dis; 2013 [cited 2021 Nov 1]. p. 907-16. Available from: https://pubmed.ncbi.nlm.nih.gov/23801600/

10. Martin MK, Venantius KB, Patricia N, Bernard K, Keith B, Allen K, et al. Correlates of uptake of optimal doses of sulfadoxine-pyrimethamine for prevention of malaria during pregnancy in East-Central Uganda. Malar $\mathrm{J}$ [Internet]. 2020 Apr 15 [cited 2021 Nov 1];19(1). Available from: https://pubmed.ncbi.nlm.nih.gov/32295601/

11. MoH. Management of cases in Ghana. J Ghana Sci. 2014;11(5):201-42.

12. WHO Global Malaria Program. New WHO recommendations for IPTp-SP [Internet]. 2013 [cited 2020 Oct 13]. Available from: http://whqlibdoc.who.int/publications/2010/9789241599412_eng.pdf

13. Azizi SC. Uptake of intermittent preventive treatment for malaria during pregnancy with SulphadoxinePyrimethamine in Malawi after adoption of updated World Health Organization policy: An analysis of demographic and health survey 2015-2016. BMC Public Health [Internet]. 2020 Mar 16 [cited 2020 Oct 18];20(1):1-12. Available from: https://link.springer.com/articles/10.1186/s12889-020-08471-5

14. De-gaulle VF, Kamgno J. Assessing Health System Factors Affecting Access and Delivery of IPTp-SP and ITN to Pregnant Women Attending ANC Clinics in Ghana-A Quantitative Study. 2020;

15. Stephen AAI, Wurapa F, Afari EA, Sackey SO, Malm KL, Nyarko KM. Factors influencing utilization of intermittent preventive treatment for pregnancy in the Gushegu district, Ghana, 2013. Pan Afr Med J. 2016;25(Supp 1):4.

16. NMCP. 1 | Page. 2021.

17. NMCP. 2017 Annual Report National Malaria Control Programme. Adv Cancer Res [Internet]. 2018; Available from: http://www.ghanahealthservice.org/downloads/NMCP_2014_ANNUAL_REPORT.pdf

18. MIS. The DHS Program - Ghana: Malaria Indicator Survey (MIS), 2019 [Internet]. 2019 [cited 2020 Oct 13]. Available from: https://www.dhsprogram.com/what-we-do/survey/survey-display-557.cfm

19. Arulogun, O.S., and Okereke CC. Arulogun, O.S., and Okereke, C.C. "Knowledge and Practices of Intermittent Preventive Treatment of Malaria in Pregnancy among Health Workers in a Southwest Local Government Area of Nigeria." Journal of Medicine and Medical Sciences 2012; 3(6): 415-422. [Internet]. 2012 [cited 2020 Oct 14]. Available from: http://www.sciepub.com/reference/57650 
20. Amankwah S, Anto F. Factors Associated with Uptake of Intermittent Preventive Treatment of Malaria in Pregnancy: A Cross-Sectional Study in Private Health Facilities in Tema Metropolis, Ghana. J Trop Med. 2019;2019.

21. Vandy AO, Peprah NY, Jerela JY, Titiati P, Manu A, Akamah J, et al. Factors influencing adherence to the new intermittent preventive treatment of malaria in pregnancy policy in Keta District of the Volta region, Ghana. BMC Pregnancy Childbirth [Internet]. 2019 Nov 20 [cited 2020 Oct 13];19(1):1-8. Available from: https://link.springer.com/articles/10.1186/s12884-019-2544-8

22. Orish VN, Onyeabor OS, Boampong JN, Afoakwah R, Nwaefuna E, Acquah S, et al. Prevalence of intermittent preventive treatment with sulphadoxine-pyrimethamine (IPTp-Sp) use during pregnancy and other associated factors in Sekondi-Takoradi, Ghana. Afr Health Sci [Internet]. 2015 Dec 1 [cited 2021 Nov 6];15(4):1087-96. Available from: https://pubmed.ncbi.nlm.nih.gov/26958008/

23. Ibrahim H, Maya ET, Issah K, Apanga PA, Bachan EG, Noora CL. Factors influencing uptake of intermittent preventive treatment of malaria in pregnancy using sulphadoxine pyrimethamine in sunyani municipality, Ghana. Pan Afr Med J [Internet]. 2017 Oct 10 [cited 2020 Oct 20];28. Available from: /pmc/articles/PMC5839217/?report=abstract

24. Oluwasomidoyin OB, Bello OO, Oni O. Health Workers ' Awareness and Knowledge of Current Recommendation of Intermittent Preventive Treatment in Pregnancy in South-Western Nigeria. 2020;

25. Arulogun OS, Okereke CC. Knowledge and practices of intermittent preventive treatment of malaria in pregnancy among health workers in a southwest local government area of Nigeria. J Med Med Sci [Internet]. 2012 [cited 2020 Oct 20];3(6):415-22. Available from:

https://www.researchgate.net/publication/274078747_Knowledge_and_practices_of_intermittent_preventive_ treatment_of_malaria_in_pregnancy_among_health_workers_in_a_southwest_local_government_area_of_Nigeria

26. Oyefabi A, Sambo M, Sabitu K. Effect of primary health care workers training on the knowledge and utilization of intermittent preventive therapy for malaria in pregnancy in Zaria, Nigeria. J Med Trop. 2015;17(1):4.

27. Maheu-Giroux M, Castro MC. Factors affecting providers' delivery of intermittent preventive treatment for malaria in pregnancy: A five-country analysis of national service provision assessment surveys. Malar $\mathrm{J}$ [Internet]. 2014 Dec 20 [cited 2020 Mar 1];13(1):440. Available from:

https://malariajournal.biomedcentral.com/articles/10.1186/1475-2875-13-440

28. Rassi C, Graham K, Mufubenga P, King R, Meier J, Gudoi SS. Assessing supply-side barriers to uptake of intermittent preventive treatment for malaria in pregnancy: A qualitative study and document and record review in two regions of Uganda. Malar J. 2016;15(1):1-16.

29. Maheu-Giroux M, Castro MC. Factors affecting providers' delivery of intermittent preventive treatment for malaria in pregnancy: A five-country analysis of national service provision assessment surveys. Malar J. 2014;13(1):1-12.

30. Mubyazi GM, Bloch P, Byskov J, Magnussen P, Bygbjerg IC, Hansen KS. Supply-related drivers of staff motivation for providing intermittent preventive treatment of malaria during pregnancy in Tanzania: Evidence from two rural districts. Malar J [Internet]. 2012 Dec 18 [cited 2020 Jan 12];11(1):48. Available from: https://malariajournal.biomedcentral.com/articles/10.1186/1475-2875-11-48

\section{Supplementary Files}

Supplements 1-3 are not available with this version. 